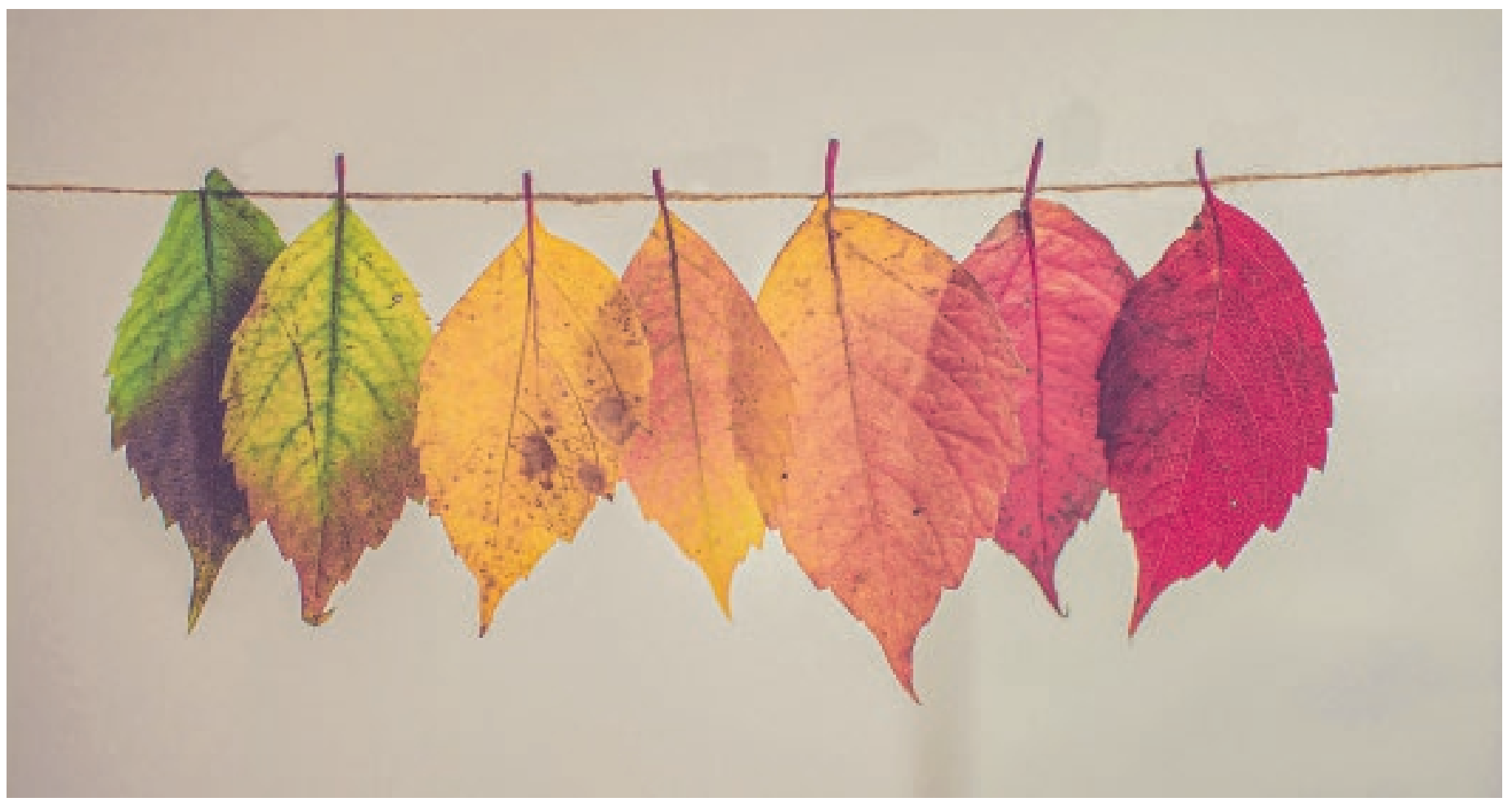

\title{
Den Blick behutsam auf das Lebensende lenken
}

\section{Hermann Amstad}

Dr. med., ehem. Generalsekretär SAMW, Basel

Palliative Care ist in der Schweiz noch nicht genügend in die Gesundheitsversorgung integriert. Das betrifft auch die frühzeitige Auseinandersetzung mit dem eigenen Lebensende und jenem der Angehörigen. Was es braucht, ist eine Sensibilisierung der Bevölkerung dafür, dass Sterben und Tod ein Teil des Lebens sind. Mit der «Palliativ-Woche '21» hat palliative bs $+b l$ dazu ein Pilotprojekt lanciert.

In der Schweiz sind die Bedeutung und die Notwendigkeit von Palliative Care seit längerem erkannt. Die Nationale Strategie Palliative Care, die Bund und Kantone sowie Fachkreise in den Jahren 2010 bis 2015 initiiert und umgesetzt haben, hat wesentliche Verbesserungen gebracht, namentlich für Patientinnen und Patienten mit onkologischen Erkrankungen.

In der Schweiz sterben jedes Jahr rund 65000 Menschen; Krebserkrankungen sind aber in weniger als einem Drittel der Fälle die Todesursache. PalliativeCare-Fachleute sind sich deshalb einig, dass die Ziele der Strategie noch nicht erreicht sind, insofern nicht alle Menschen Zugang zu Palliative Care haben. Dies hat die Kommission Soziale Sicherheit und Gesundheit des Ständerates 2018 veranlasst, den Bundesrat in einem Postulat aufzufordern, eine Standortbestimmung vorzunehmen und mögliche Verbesserungsmassnahmen zu skizzieren [1].

\section{Palliative Care: Noch gibt es Lücken}

Für die Beantwortung des Postulats wurde in einem ersten Schritt eine Situationsanalyse durchgeführt; diese hat vier Problembereiche identifiziert: 
1. Mit den heutigen Strukturen im Gesundheitswesen wird es nicht möglich sein, die zunehmende Anzahl sterbender Menschen und ihrer Angehörigen angemessen zu behandeln und zu betreuen.

2. Das Lebensende wird als akutes Ereignis behandeltobwohl rund 70\% der Todesfälle in der Schweiz absehbar sind und damit frühzeitig und vorausschauend vorbereitet werden könnten.

3. Die gesellschaftliche Entwicklung hin zu einem «gestaltbaren» und «selbstbestimmten Sterben» führt zu neuen Ungleichheiten am Lebensende.

4. Angebote der Palliative Care sind nicht ausreichend in die Gesundheitsversorgung integriert; nicht alle Patientengruppen haben den gleichen Zugang zu diesen Angeboten.

Im Bericht zum Postulat, der im Herbst 2020 veröffentlicht wurde, nennt der Bundesrat zahlreiche Verbesserungsmassnahmen. Gleichzeitig kommt der Bundesrat zum Schluss, dass es zwingend notwendig sei, Sterben und Tod als Bestandteile des Lebens und der Gesundheitsversorgung anzuerkennen. Dazu soll-

Das Ziel des Projektes besteht darin, die Öffentlichkeit dafür zu sensibilisieren, dass zu einem guten Leben auch ein gutes Sterben gehört.

ten Rahmenbedingungen geschaffen werden, damit sich alle Menschen frühzeitig mit ihrem Lebensende auseinandersetzen können. Insbesondere gehe es darum, Diskussionen und Gespräche über persönliche Wünsche, Erwartungen und Vorstellungen anzuregen und gegebenenfalls festzuhalten; ausserdem sei es wichtig, dass die Angebote der Palliative Care bekannt seien [2].

\section{Die «Palliativ-Woche '21» als Pilotprojekt}

Wie die Sensibilisierung und die Information der Bevölkerung zu gestalten sind, lässt der Bericht des Bundesrates offen. Ideal wäre wohl eine grossangelegte, d.h. schweizweite und mehrjährige Kampagne, wie dies im HIV-Bereich oder im Rahmen der Covid-19-Pandemie geschehen ist. Da sich der Bund dafür nicht zuständig sieht, hat der Verein palliative bs + bl (eine Sektion von palliative ch in den Kantonen Basel-Stadt und Baselland) beschlossen, vorerst auf regionaler Ebene - auch im Sinne eines Pilotprojekts - eine solche Kampagne zu lancieren. Von 2021 bis 2025 wird jährlich eine thematische Woche "Palliative Care» (oder kurz: PalliativWoche) durchgeführt. Es werden nacheinander die Themen «Lebensqualität bis zuletzt», «Die verbleibende Lebenszeit gestalten», "Gemeinsam statt einsam», «Palliative Care: wie, wann und wo?» und «Palliative Care was bleibt zu tun?» behandelt. Das Ziel dieses Projektes besteht darin, eine breite Öffentlichkeit dafür zu sensibilisieren,

- dass zu einem guten Leben auch ein gutes Sterben gehört und daher die Lebensqualität bis zum Schluss wichtig ist;

- dass Palliative Care nicht bedeutet, «jemanden aufzugeben", sondern vielmehr, in der letzten Lebensphase (die mehrere Monate oder sogar Jahre dauern kann) den Blick auf die Erhaltung einer guten Lebensqualität zu richten.

In den Kantonen Basel-Stadt und Baselland gibt es zahlreiche Institutionen bzw. Organisationen, die sich in der einen oder anderen Form mit dem Lebensende befassen; von diesen haben sich GGG Voluntas, Curaviva Basel-Stadt, Curaviva Baselland, Spitex Basel, die Kommission Palliative Care der Landeskirchen Basel-Stadt,

\footnotetext{
Aktionen während der Palliativ-Woche '21

Die Vorstellungen vom guten Sterben sind sehr persönlich. Was Lebensqualität am Lebensende wirklich heisst, variiert von Mensch zu Mensch. Die Palliativ-Woche '21 lädt ein, sich Gedanken darüber zu machen.

Im Rahmen von Theater- und Filmaufführungen, Erzählrunden, Referaten und Podiumsdiskussionen in Basel, Riehen, Allschwil, Münchenstein, Therwil, Sissach und Liestal besteht die Gelegenheit, sich mit der Frage "Was zählt, wenn die Tage gezählt sind?» auseinanderzusetzen.

Zusätzlich werden in der Region grosse und kleine Plakate angebracht. Sie formulieren möglich Antworten, z.B. «Rückschau halten?», "Schmerzfrei sein?» oder "Zuhause bleiben?». Jeder und jede ist eingeladen, für sich die zutreffende Antwort zu finden. Ausserdem gibt es eine Website (palliativ-woche.ch), auf der das Programm der Palliativ-Woche sowie Informationen zu Palliative Care zu finden sind.
}

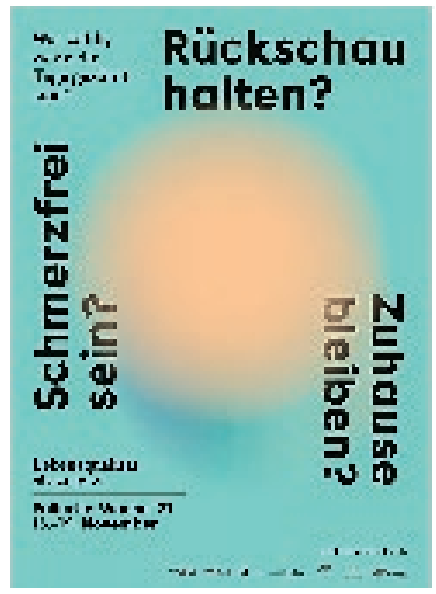

(C) palliative bs + bl 
die Ökumenische Koordinationsstelle Palliative Care Baselland sowie Pro Senectute beider Basel bereit erklärt, in der Trägerschaft der Palliativ-Woche mitzuwirken und einen Beitrag zu leisten, und zwar sowohl fi-

\section{Sterben und Tod sind in der Gesellschaft immer noch tabuisiert. Als Folge ist das Verständnis von und für Palliative Care bescheiden.}

nanziell als auch durch eigene Aktivitäten. Es ist davon auszugehen, dass diese Organisationen in ihrer Funktion als Multiplikatoren die Wirkung der Palliativ-Woche markant erhöhen werden.

\section{Sensibilisierung als Grundlage}

Sterben und Tod sind in der Gesellschaft immer noch mehrheitlich tabuisiert. Eine Folge davon ist, dass das Verständnis von und für Palliative Care in der Bevölkerung bescheiden ist. Palliative-Care-Fachleute teilen die Ansicht des Bundesrates, dass ein gemeinsames

\section{Das Wichtigste in Kürze}

- Die Angebote der Palliative Care sind noch nicht ausreichend in die Gesundheitsversorgung integriert. Dies liegt zum Teil daran, dass Sterben und Tod in der Gesellschaft immer noch tabuisiert sind.

- Der Verein palliative bs+bl lanciert von 2021 bis 2025 die Kampagne "Palliativ-Woche», um die Bevölkerung für die Palliative Care zu sensibilisieren.

- Ein breites Verständnis von und für Palliative Care, bei dem die Lebensqualität in der letzten Lebensphase im Zentrum steht, soll die Grundlage für weitere Verbesserungsmassnahmen bilden. und breites Verständnis von und für Palliative Care in der Bevölkerung, bei dem nicht primär die Sterbephase, sondern die Lebensqualität in der letzten Lebensphase im Zentrum steht, die Grundlage bildet für alle weiteren Verbesserungsmassnahmen: Erst auf dieser Basis sind die Menschen bereit, sich rechtzeitig mit dem eigenen Sterben und Tod auseinanderzusetzen, und steigt die Nachfrage nach Palliative Care. Und erst auf dieser Basis entsteht schliesslich auch der Druck auf die Politik, die fehlenden Angebotsstrukturen zu schaffen und die immer noch offenen finanziellen Fragen befriedigend zu lösen.

\section{Bildnachweis}

Chris Lawton / Unsplash

\section{Literatur}

1 Kommission für soziale Sicherheit und Gesundheit des Ständerates (SGK-SR). Bessere Betreuung und Behandlung von Menschen am Lebensende. Postulat 18.3384

2 Bundesrat. Bessere Betreuung und Behandlung von Menschen am Lebensende. Bericht in Erfüllung des Postulates 18.3384 der SGKSR.

\section{L'essentiel en bref}

- Les services de soins palliatifs ne sont pas encore suffisamment intégrés dans les soins de santé. Cela s'explique en partie par le fait que le décès et la mort sont encore tabous dans la société.

- L'association palliative bs+bl lance la campagne "Semaine des soins palliatifs» de 2021 à 2025 pour sensibiliser la population aux soins palliatifs.

- Une large compréhension de et pour les soins palliatifs, qui mettent l'accent sur la qualité de vie dans la dernière phase de la vie, constitue la base de nouvelles mesures d'amélioration. 